\title{
El Vaticano II, ¿̨texto constitucional de la fe? Una carta de Peter Hünermann
}

\section{INTRODUCCIÓN}

\section{CARLOS SCHICKENDANTZ}

En 2015 se publicó en la revista un trabajo de mi autoría sobre una propuesta hecha por Peter Hünermann referida al género literario del Vaticano II: caracterizar al texto conciliar con el concepto de constitución proveniente del ámbito de las ciencias sociales ${ }^{1}$. El autor alemán, uno de los mayores especialistas internacionales sobre el Concilio, había publicado en 2006 un trabajo muy exhaustivo en el marco del importante Comentario editado por Herder ${ }^{2}$.

Naturalmente que el corazón de su aporte está dado por el resultado al que se arriba: el Vaticano II como texto constitucional de la fe con sus consecuencias eclesiales y hermenéuticas. Pero, como se expresaba en el artículo de Teología y Vida, lo que también otorga valor a esa contribución reside en la hermenéutica del texto que se delinea, en la consistencia del largo y ordenado camino que se recorre y en el minucioso análisis que se realiza a los más diversos componentes -discursos, decisiones, modos de trabajo, estructura de los documentos- para que el texto conciliar se explique a sí mismo -el despliegue del mundo del texto inseparable de su actualización en la lectura- y la analogía de "texto constitucional" resulte plausible.

\footnotetext{
C. Schickendantz, "El Vaticano II como texto constitucional de la fe. Valor y límites de una interpretación del género textual”, en Teología y Vida 56 (2015) 229-259.

2 Cf. P. Hünermann, "Der Text: Werden - Gestalt - Bedeutung. Eine hermeneutische Reflexion", en P. Hünermann (ed.), Herders Theologischer Kommentar zum zweiten Vatikanischen Konzil 5. Theologische Zusammenschau und Perspektiven (Herder, Freiburg i.Br. 2006) 1-95. Existe un texto breve próximo, pero con varias diferencias: P. Hünermann, "El 'texto' pasado por alto. Sobre la hermenéutica del concilio Vaticano II”, en Concilium 312 (2005) 139-160.
} 
El artículo ponía de relieve, también, ideas centrales de la significativa investigación del teólogo del Centre Sèvre de París, Christoph Theobald, en particular, de su trabajo más importante de $2009^{3}$. Al momento de entregar el artículo para su posible publicación, todavía no había aparecido un nuevo libro de Theobald sobre el asunto: Le concile Vatican II. Quel avenir?, Cerf, Paris 2015.

Si bien Hünermann y Theobald comparten una perspectiva y sensibilidad común en el panorama de la teología contemporánea, que incluso se verifica en que ambos han tomado parte en los dos trabajos editoriales más relevantes que hoy existen sobre el Vaticano II -la Historia dirigida por G. Alberigo y el Comentario editado por Hünermann y Hilberathrepresentan en este aspecto específico -la analogía constitucional- puntos de vista diversos. Recoger el planteo central de Theobald, con múltiples notas a lo largo del artículo y, sobre todo, explicitar el núcleo de su perspectiva hermenéutica ofrecía dos ventajas: por una parte, sacar a la luz una de las lecturas más fundadas y creativas en el panorama internacional sobre el Vaticano II, por otra, poner de relieve de la mejor manera las limitaciones que ofrece la analogía constitucional. De allí la breve conclusión que se formulaba: dicha analogía permanece como un aporte valioso, pero limitado.

En su nuevo libro de 2015 recién citado -Le concile Vatican II. Quel avenir?-, posterior al artículo de Teología y Vida, C. Theobald ha vuelto a repetir, varias veces, las observaciones críticas a esta analogía de Hünermann ${ }^{4}$. No parece que haya argumentos nuevos en relación a su trabajo de 2009, sintetizados en las páginas 255-258 del artículo.

Creo que la crítica de Theobald puede condensarse en tres puntos. 1) La analogía constitucional favorece una concentración del proceso de recepción posconciliar en la eclesiología -la interpretación Suenens-Pablo VI del Concilio- y no en la perspectiva hermenéutica, "pastoral", como recepción de la Escritura -o reinterpretación de la Tradición- en nuevos y variados contextos culturales, DV y GS, -la interpretación Juan XXIII-. En este sentido, la explicación de Hünermann le afecta a Theobald en el núcleo de su interpretación del Va-

3 C. Theobald, La réception du concile Vatican II. 1. Accéder à la source (Cerf, Paris 2009). Está pendiente la publicación del segundo volumen que, según información del mismo autor, recién se haría en 2017.

4 Particularmente en las páginas 41-42, 45, 59, 110-113, 129, 139-140, 156-157. 
ticano II. 2) Creo que Theobald no advierte que, para Hünermann, la analogía es un elemento, una perspectiva entre otras posibles; no representa la clave de interpretación, sino un abordaje más. De hecho, a diferencia de otros teólogos y teólogas, el autor posee varios aportes sobre la lectura del Concilio5. 3) Además, Theobald entiende la analogía solo o primordialmente como una interpretación jurídica y, por tanto, juzga que la lectura del teólogo de Tubinga no pondría suficientemente de relieve la transformación de lo "dogmático", de lo "doctrinal" que supone la pastoralidad del Concilio.

No es posible aquí un análisis más detallado. En el artículo afirmé que varias de las preocupaciones de Theobald referidas son compartidas por Hünermann en otros textos. En ese sentido, interpreto las reservas de Theobald como "advertencias saludables" - expresión que se utiliza en el lenguaje ecuménico-, pero ellas no serían directamente aplicables a la forma como Hünermann comprende la analogía constitucional. En cambio, la preocupación fundamental del teólogo de París me parece acertada: esta interpretación -si se la considera aisladamente- confirma una lectura unilateralmente eclesiológica del Concilio y del proceso de recepción.

Al leer P. Hünermann el artículo de Teología y Vida, me hizo llegar una carta personal en la que explicita más su opción de sostener las bondades de dicha analogía como una aproximación que, junto a otras, ayuda a descubrir la riqueza y explicar el valor del Vaticano II para la vida de la Iglesia. Como podrá observarse, Hünermann cita las observaciones críticas de Theobald a partir del artículo y no de la obra misma del teólogo de París. Con el deseo de servir al diálogo teológico, el director de la revista decidió, con las consultas pertinentes y el consentimiento del autor, ofrecer aquí en alemán y en castellano la carta de Hünermann.

\section{CARTA DE P. HÜNERMANN}

Querido Carlos Schickendantz,

He leído con gran interés el artículo en Teología y Vida 3 (2015) 229-259. En particular también las objeciones que Christoph Theobald expone

5 Hemos ofrecido al público de lengua castellana una selección y traducción de algunas de dichas contribuciones. Cf. P. Hünermann, El Vaticano II como software de la Iglesia actual (Ed. Universidad Alberto Hurtado, Santiago 2014). 
contra mi posición. Quisiera contribuir con algunas ideas a la clarificación del asunto.

Una primera y muy fundamental observación: en el tomo quinto del comentario al Concilio Vaticano II he llamado expresamente la atención, así lo creo, que la pregunta por el texto representa un elemento en la discusión actual acerca de las perspectivas hermenéuticas en las que el Concilio Vaticano II se debe interpretar hoy. Parto de que no se puede presentar un sistema cerrado de perspectivas hermenéuticas. El desarrollo del planteo hermenéutico, por ejemplo, a partir de Schleiermacher, muestra esto claramente. Son momentos diferentes que deben ser tenidos en cuenta.

¿Por qué, entonces, la cuestión de la peculiaridad del texto en este modo formal tal como yo lo he hecho? Este tema se plantea hoy en la filología y se valora como una importante perspectiva hermenéutica. La particularidad de estos momentos diferentes del planteo hermenéutico se fundamenta en que aquí cada momento entrelaza, co-determinando, las otras dimensiones de la hermenéutica.

Esta cuestión, sin embargo, no se plantea en la teología, sobre todo en la teología sistemática, aunque se vislumbran claramente en la investigación de la historia del dogma profundos cambios en la forma de los textos magisteriales. En la Neoescolástica y en el Vaticano I, el foco teológico está concentrado en definiciones magisteriales, es decir, en anatemas. Por medio de ellos, se condenan las herejías; se definen los límites. La "doctrina" añadida con la referencia a la tradición, las citas de anteriores declaraciones magisteriales, etc., tienen solamente una función de ayuda explicativa; ofrecen ayuda a la comprensión, más no. Estas exposiciones representan un cierto desarrollo ulterior en la praxis de Trento. Allí el acento está puesto de un modo más exclusivo en los anatemas. Las condenas atañen a las posiciones centrales de los planteos de los reformadores. La doctrina falta de modo considerable, se la presupone como ya dada y como conocida en general, y solo los "modernizadores" en el episcopado orientados según la visión de Erasmo adaptaron esta doctrina para cada decreto. Entre otras razones, con el fundamento de que los párrocos no sabían en absoluto lo que en realidad significaba la doctrina de la justificación. En los predecesores a Trento se ve claramente que, en las formulaciones de la respectiva doctrina, ni con mucho obraba el cuidado que se empleaba para formular anatemas. Me doy por satisfecho aquí con estas pocas indicaciones para mostrar cómo en el Concilio Vaticano II se llevó a cabo un giro decisivo. 
El Vaticano II, ¿texto constitucional de la fe? Una carta de Peter Hünermann $\mid 121$

Cambia el lenguaje magisterial. Surge un nuevo modelo perfeccionado de doctrina. No se trata de condenas y delimitaciones de herejías. Se pule la doctrina. ¿Por qué? A raíz de otras relaciones sociales, de nuevos accesos y conocimientos en el marco de la historia, de la naturaleza, de nuevos métodos científicos, etc., se requiere de un fundamentado aggiornamento de la doctrina, esto es del hablar -del testimoniar- del misterio de la fe. En mis reflexiones se trató de la determinación de este tipo de texto. Este tipo de texto contiene evidentemente elementos estilísticos diferenciados. No define, sino que expresa un modo de hablar formalmente "histórico", un modo de hablar que narra, pero un modo que al mismo tiempo tiene también un carácter vinculante $y$, naturalmente, una plausibilidad, es decir, un modo que procura un hablar fundamentado, un hablar que, además, está caracterizado por su carácter de respuesta al obrar divino.

Si Christoph Theobald ve una analogía con la Biblia (cf. p. 257), entonces se trata de una analogía-afirmación llena de sentido, pero que no puede fácilmente estar fundamentada por medio de referencias a esta característica estilística. El "Nuevo Testamento" representa el documento constituyente, el acta fundacional de la fe cristiana, de la comunidad cristiana de fe. (No entro ahora más detalladamente en la insoluble pertenencia mutua de la Biblia en el sentido del Antiguo y del Nuevo Testamento.)

La pregunta entonces se formula así: ¡cómo se relaciona el corpus de textos del Concilio Vaticano II con un documento constituyente, con un acta fundacional? Si aquí se elige la expresión "texto constitucional", no es para poner el corpus de textos del Vaticano II en lugar del documento fundacional, sino para expresar cómo le es dado al documento fundacional una forma, una expresión que la hace revivir en la nueva situación histórica. Este es el fundamento de la analogía.

En alemán y en inglés se utiliza el sustantivo "constitución", como el correspondiente adjetivo, tanto para la "constitución" de una persona, de una cosa, como también en el ámbito político y jurídico para la constitución de un Estado. Constitución es, entonces, un concepto general que no significa solo una característica única sino el todo de la cosa: su estado. El estado puede variar, aunque no rápida o incidentalmente. El estado afecta a la cosa en su totalidad. Constitución expresa además un texto - por ejemplo, una ley, una ordenanza, un decreto papal, resolucio- 
nes conciliares- por medio del cual un hecho histórico se determina y/o se clasifica autoritativamente en su constitución ${ }^{6}$.

Esta ampliación del significado corresponde al latín: Karl Ernst Georges (Ausführliches lateinisch-deutsches Handwörterbuch, aus den Quellen zusammengetragen... Mannheim 1960 11. Auflage) indica como posibilidades de traducción para el sustantivo:

I. Disposición firme, constitución:

a) Disposición física, condición, estado de una cosa;

b) Institución política, constitución (por ejemplo, Cicero, constitutio religionum, rei publicae).

II. Declaración, prescripción:

a) Definición explicativa o conceptual;

b) Justificación o modo de fundamentación en caso de litigio;

c) Disposición u ordenanza de una autoridad pública.

En francés, Langenscheidt ofrece como traducción del sustantivo "constitution" igualmente un doble significado, pero para el adjetivo trae solamente el significado político-jurídico, aparte del lenguaje técnico de la medicina que muestra con esto los diagnósticos de enfermedades de nacimiento.

En español, según la información del diccionario de la lengua española de la Real Academia Española, 19. Edición, Madrid 1970, me parece similar como en el francés.

Desde aquí respondo entonces a los diferentes reproches críticos, 257ss.: el término "texto constitucional" fue elegido porque "constitucional", a partir de su triple significación, ofreció la posibilidad de reflexionar un tipo de texto que es singular. ¿En qué consiste esta singularidad? Los textos contienen, por un lado, un carácter público vinculante para la Iglesia como una comunidad fundada que tiene una figura o "constitución" histórica cambiante. (Theobald habla expresamente de la "situación inédita" en la que la Iglesia ha entrado ahora.) Estos textos contienen, por consecuencia, un momento jurídico sin ser de naturaleza jurídica ${ }^{7}$. Este

\footnotetext{
Cf. Der große Duden, Bd. 5, Mannheim 1960.

7 En mi exposición se rechaza expresamente la mera comprensión jurídica.
} 
corpus constitucional tiene -visto formalmente- la peculiaridad de fijar solamente sentidos $\mathrm{u}$ orientaciones fundamentales para la acción, no de prescribir, por ejemplo, una legislación básica: los textos tematizan contenidos fundamentales de la doctrina de la fe en el lenguaje pastoral de hoy y conforme con modos de comprensión y mentalidades actuales, sin cuya aceptación un discurso actual de la Revelación, de la Iglesia y de la Iglesia en el mundo o de la liturgia no puede estar conforme con la fe de la Iglesia. En este sentido, los textos del Concilio reflejan las líneas-base de la ratio fidei en relación a los misterios centrales de la fe y, sin embargo, en un modo testimonial y correspondiente a la comprensión general y a la pastoral, es decir, a la edificación en la fe. Esto se expresa en forma concreta: "la mundialización, un pluralismo cultural insuperable y un estatuto de diáspora impensable para el cristianismo del segundo milenio" (p. 258). Pero porque el corpus textual tiene naturaleza constitucional, es decir, porque exige de modo necesario actualizaciones como punto de partida, esta expresión produce precisamente eso que Theobald ve amenazado por medio de la misma expresión, es decir, "la necesaria historización de los procesos de conversión y de aprendizaje requeridos".

Con estas aclaraciones no quisiera de ninguna manera desacreditar el trabajo de Christoph Theobald. Sus reflexiones ofrecen planteos muy ricos para la configuración de la teología actual y le estoy agradecido por eso.

Espero que, por medio de esta carta, mi intención y también la elección de la palabra "constitucional" hayan ofrecido la explicación deseada.

Para finalizar, una observación histórica.

En el pontificado de Benedicto XVI y ya en su tiempo como prefecto de la Congregación de la fe, ganó terreno cada vez más una interpretación del Concilio Vaticano II que, por ejemplo, estuvo representada por Cardenales como Brandmüller o el Arzobispo Marquetto ${ }^{8}$. Precisamente frente a tales tendencias no débiles ${ }^{9}$, me interesó destacar que los textos del Concilio Vaticano II no deben ser comprendidos a partir de las distinciones pasadas entre definiciones dogmáticas y aplicaciones

8 Cf. G. Miccoli, La Chiesa del anticoncilio. I traditionalisti a la riconquista di Roma, Roma-Bari 2011.

9 Cf. Roberto de Mattei, Das Zweite Vatikanische Konzil. Eine bislang ungeschriebene Geschichte. Ruppichteroth: Edition Kirchliche Umschauf 2011. 
pastorales. Se trata realmente de un nuevo tipo de textos para formular la doctrina vinculante de la Iglesia y de un modo que exige necesarias actualizaciones y concretizaciones.

El proceso del Sínodo recién concluido ha mostrado cuán necesarios son los textos constitucionales.

El largo debate de Benedicto XVI con el movimiento de Lefebvre, su disputa en relación a la continuidad y discontinuidad en la comprensión del Concilio, su preocupación por el texto del Concilio contra un espíritu del Concilio aparentemente vago, está relacionado, según mi perspectiva, con una cierta perplejidad en relación a la peculiaridad de este nuevo tipo de texto en el que se expresa el carácter único de la Iglesia, de la comunidad de los creyentes, del Pueblo de Dios, del Cuerpo de Cristo, que al mismo tiempo "ut societas constituta et ordinata subsistit in Ecclesia catholica".

Después de esta larga carta, me despido con un saludo cordial.

Peter Hünermann

VERSIÓN ORIGINAL:

Prof. Dr. Peter Hünermann Engwiesenstr. 14

72108 Rottenburg-Oberndorf Datei: Brief.Carlos.Schickendantz.8.11.15

Lieber Carlos Schickendantz,

ich habe den Artikel in Teología y Vida 3 (2015) 229-259 mit großem Interesse gelesen. Insbesondere auch noch einmal die Einwände, die Christoph Theobald gegen meine Position vorbringt. Ich möchte zur Abklärung einige Gedanken beisteuern.

Eine erste, sehr grundsätzliche Anmerkung: Ich habe -so meine ichim Band 5 des Kommentars zum II. Vatikanum ausdrücklich darauf aufmerksam gemacht, dass die Frage nach dem Text ein Element in der gängigen Diskussion um die hermeneutischen Perspektiven darstellt, in denen das II. Vatikanische Konzil heute zu interpretieren ist. Ich gehe davon aus, dass man kein abgeschlossenes System hermeneutischer Perspektiven vorlegen kann. Die Entwicklung der hermeneutischen Fra- 
El Vaticano II, ¿texto constitucional de la fe? Una carta de Peter Hünermann $\mid 125$

gestellung, etwa von Schleiermacher ab, erweist dies deutlich. Es sind unterschiedliche Momente, die berücksichtigt werden müssen.

Warum also die Frage nach der Eigenart des Textes in dieser formalen Art, wie ich es getan habe? Diese Frage wird heute in der Philologie gestellt und wird als eine wichtige hermeneutische Perspektive gewürdigt. Die Eigentümlichkeit dieser verschiedenen Momente der hermeneutischen Fragestellung, beruht ja darauf, dass hier jeweils die einzelnen Momente die anderen Dimensionen der Hermeneutik mit-bestimmend durchwirken.

Diese Frage wird aber in der Theologie, vor allem aber in der systematischen Theologe so nicht gestellt, obwohl sich durch die dogmengeschichtliche Forschung deutlich tiefgreifende Veränderungen in lehramtlichen Textformen abzeichnen. In der Neuscholastik und im I. Vatikanum ist der theologische Blick völlig auf die lehramtlichen Definitionen, d. h. auf die Anathematismen konzentriert. Durch Anathematismen werden Häresien verurteilt. Es werden Abgrenzungen definiert. Die beigefügte „Doctrina“ mit den Verweisen auf die Tradition, die Zitationen früherer lehramtlicher Äußerungen etc. haben lediglich eine erläuternde Hilfsfunktion. Sie bieten Verständnishilfen, nicht mehr. Diese Ausführungen stellen eine gewisse Fortentwicklung der Praxis in Trient dar. Dort liegt der Akzent noch viel exklusiver auf den Anathematismen. Die Verwerfungen betreffen Kernpositionen der reformatorischen Positionen. Die Doctrina fehlt weitgehend, sie wird als gegeben und allgemein bekannt vorausgesetzt und lediglich die erasmianisch orientierten „Modernisierer“ im Episkopat setzen jeweils für einzelne Dekrete diese Doctrina durch. Unter anderem mit der Begründung, dass ja die Pfarrer gar nicht wüßten, was die Rechtfertigungslehre eigentlich meine. Man sieht an den Vorgängen in Trient deutlich, dass bei der Formulierung der jeweiligen Doctrina bei weitem nicht die Sorgfalt waltete, die man aufwandte, um die Anathematismen $\mathrm{zu}$ formulieren. Ich begnüge mich hier mit diesen wenigen Hinweisen, um aufzuzeigen, wie sich im II. Vatikanischen Konzil eine entscheidende Veränderung vollzieht. Es verändert sich das lehramtliche Sprechen. Es entsteht ein neuer fortgebildeter Typ von Doctrina. Es geht nicht um Verwerfungen und Abgrenzung von Häresien. Es wird an der Doctrina gefeilt. Warum? Es braucht aufgrund anderer gesellschaftlicher Verhältnisse, neuer Zugänge und Einsichten in die Geschichte, in die Natur, neuer wissenschaftlicher Methoden etc. etc. eines grundlegenden aggiornamento 
der Doctrina, d. h. des Sprechens -von und des Bezeugens- der Mysterien des Glaubens. In meinen Ausführungen ging es mir um die Bestimmung dieses Texttypus. Dieser Texttypus umschließt selbstverständlich unterschiedliche stilistische Elemente. Er definiert nicht, sondern spricht eine erzählende, eine formal "geschichtliche" Sprechweise, eine Sprechweise aber, die zugleich auch einen verpflichtenden Charakter hat und natürlich eine Plausibilität, d. h. um ein begründetes Sprechen bemüht ist, ein Sprechen, das darüber hinaus jeweils durch seinen Antwortcharakter auf göttliches Handeln charakterisiert ist.

Wenn Christoph Theobald eine Analogie zur Bibel sieht (vgl. S. 257), dann ist eine solche Analogie-Aussage sinnvoll, kann aber nicht einfach durch Verweise auf diese stilistischen Merkmale begründet werden. Das „Neue Testament" stellt die Gründungsurkunde, die Stiftungsurkunde des christlichen Glaubens, der christlichen Glaubensgemeinschaft dar. (Ich gehe jetzt nicht näher auf die unlösliche Zusammengehörigkeit von Bibel, im Sinne des Alten Testaments und des Neuen Testaments, ein.)

Die Frage lautet dann: Wie verhält sich das Textcorpus des II. Vatikanischen Konzils zur Grüdnungs- oder Stiftungsurkunde. Wenn hier der Ausdruck „konstitutioneller Text“ gewählt wird, so nicht um an die Stelle der Stiftungsurkunde das Corpus der Texte des II. Vatikanums treten zu lassen, sondern um auszudrücken, wie der Stiftung eine Gestalt bzw. ein Ausdruck zu geben ist, die sie in der neuen geschichtlichen Situation aufleben lässt. Dies ist der Grund der Analogie.

Man gebraucht im Deutschen wie im Englischen das Substantiv „Konstitution“ wie das entsprechende Adjektiv sowohl für die „Verfassung" eines Menschen, einer Sache, wie auch im politisch-juridischen Bereich für die Verfassung eines Staates. Verfassung ist so ein genereller Begriff, der nicht nur eine einzelne Eigenschaft meint, sondern das Ganze der Sache: ihren Zustand. Der Zustand kann sich ändern, aber nicht schnell, beiläufig. Er betrifft die Sache im Ganzen. Darüber hinaus bezeichnet Verfassung einen Text -etwa eine Rechtsbestimmung, Anordnung, päpstliche Erlasse, Konzilsbeschlüsse-, durch welche ein geschichtlicher Sachverhalt in seiner Verfassung autoritativ geordnet und/oder festgelegt wird ${ }^{10}$.

$10 \quad$ Vgl. Der große Duden, Bd. 5, Mannheim 1960. 
El Vaticano II, ¿texto constitucional de la fe? Una carta de Peter Hünermann $\mid 127$

Diese Bedeutungsbreite entspricht dem Lateinischen: Karl Ernst Georges, Ausführliches lateinisch-deutsches Handwörterbuch, aus den Quellen zusammen getragen... Mannheim 1960 11. Auflage gibt für das Substantiv als Übersetzungsmöglichkeiten vor:

I. Die feste Einrichtung, Verfassung:

a) die physische Einrichtung, Beschaffenheit, Zustand einer Sache;

b) Politische Einrichtung, Verfassung (z. B. Cicero, constitutio religionum, rei publicae).

II. Die Feststellung, Bestimmung:

a) die erklärende oder definierende Begriffsbestimmung;

b) Begründung und Begründungsform im Streitfall;

c) Verfügung, Anordnung einer öffentlichen Autorität.

Im Französischen bietet Langenscheidt zur Übersetzung des Substantivs „Constitution“ ebenfalls eine doppelte Bedeutung an, für das Adjektiv aber bringt er lediglich die politisch-juridische Bedeutung, abgesehen von der medizinischen Fachsprache, die damit angeborene Krankheitsbefunde bezeichnet.

Im Spanischen scheint mir dies nach Auskunft des Diccionario de la lengua española der Real Academia Española, 19. Ausgabe, Madrid 1970, ähnlich wie im Französischen zu sein.

Von daher antworte ich so auf die verschiedenen kritischen Einwände, 257f.: Der Terminus „konstitutionelle Texte“ wurde gewählt, weil „konstitutional - konstitutionell“, von seiner dreifachen Bedeutung her, die Möglichkeit bot, einen Typus von Texten zu bedenken, der einzigartig ist. Worin besteht die Einzigartigkeit? Die Texte tragen auf der einen Seite einen verpflichtenden öffentlichen Charakter für die Kirche als eine gestiftete Gemeinschaft, die wechselnde geschichtliche Gestalten oder ,Verfassungen' hat. (Theobald spricht ausdrücklich von der „situación inédita“, in die die Kirche jetzt eingetreten ist.) Diese Texte umschließen folglich ein juridisches Moment, ohne juridischer Natur zu $\operatorname{sein}^{11}$. Dieses konstitutionelle Textcorpus hat -formal gesehen- die Eigenart, lediglich grundlegende Handlungsori-

11 Es wird in meiner Darstellung ausdrücklich das rein juristische Verständnis zurückgewiesen. 
entierungen bzw. Verständnisse vorzugeben, nicht etwa eine Rahmengesetzgebung vorzuschreiben: Die Texte thematisieren grundlegende Sachverhalte der Glaubenslehre in heutiger pastoraler Sprache und konform mit heutigen Verstehensformen und Denkweisen ohne deren Akzeptanz eine heutige Rede von der Offenbarung, von der Kirche bzw. von der Kirche der Welt oder der Liturgie mit dem Glauben der Kirche nicht übereinstimmen könnte. In diesem Sinn spiegeln die Texte des Konzils die Basis-Linien der ratio fidei in Bezug auf zentrale Mysterien des Glaubens, und zwar in einer bezeugenden, dem Allgemeinverständnis und der Pastoral, d. h. der Auferbauung im Glauben entsprechenden Weise. Es drückt sich darin in konkreter Form „la mundalisación, un pluralismo cultural insuperable y un estatuto de diáspora impensable para el cristinanismo del segundo milenio" aus (p. 258). Weil das Textcorpus aber konstitutioneller Natur ist, d. h. notwendiger Weise als Ausgangspunkt Fortschreibungen fordert, leistet dieser Ausdruck gerade das, was Theobald durch den Ausdruck bedroht sieht, nämlich „la necesaria historización de los procesos de conversión y de aprendizaje requeridos“.

Mit diesen Erläuterungen möchte ich in keiner Weise die Arbeit von Christoph Theobald herabsetzen. Seine Überlegungen bieten sehr fruchtbare Ansätze für die Gestaltung der heutigen Theologie und ich bin ihm dankbar dafür.

Ich hoffe, dass durch diesen Brief meine Intention und auch die Wahl des Wortes „konstitutional“- „konstitutionell“ die wünschenswerte Erläuterung erfahren haben.

Zum Abschluss noch eine historische Anmerkung.

Im Pontifikat Benedikts XVI. und bereits in seiner Zeit als Präfekt der Glaubenskongregation gewann eine Interpretation des II. Vatikanischen Konzils immer mehr an Boden, die etwa durch Kardinäle wie Brandmüller oder Erzbischof Marquetto repräsentiert wurde ${ }^{12}$. Gerade gegenüber solchen weiß Gott nicht schwachen Tendenzen ${ }^{13}$,c kam es mir darauf an, dass die Texte des II. Vatikanischen Konzils nicht von der früheren Unterscheidung Dogmatische Definitionen - Pastorale Anweisungen her zu ver-

12 Cf. G. Miccoli, La Chiesa del anticoncilio. I traditionalisti a la riconquista di Roma, Roma-Bari 2011.

13 Vgl. Roberto de Mattei, Das Zweite Vatikanische Konzil. Eine bislang ungeschriebene Geschichte. Ruppichteroth: Edition Kirchliche Umschauf 2011. 
stehen sind. Es geht wirklich um einen neuen Typus von Texten, die Doctrina der Kirche, die verpflichtend ist, zu formulieren, und in einer Weise, die die notwendige Fortschreibung und Konkretisierung einfordert.

Der Verlauf, der gerade abgeschlossenen Synode hat gezeigt, wie notwendig konstitutionelle Texte sind.

Die jahrelange Auseinandersetzung Benedikts XVI. mit der Bewegung von Lefebvre, sein Ringen um die Kontinuität und Diskontinuität im Verständnis des Konzils, sein Mühen um den Text des Konzils gegen einen scheinbar frei schweifenden Geist des Konzils hängen m. E. mit einer gewissen Sprachlosigkeit in Bezug auf die Eigenart diese neuen Texttypus zusammen, in dem sich der einzigartige Charakter der Kirche, der Gemeinschaft der Glaubenden, dem Volk Gottes, dem Leib Christi ausspricht, die zugleich „ut societas constituta et ordinata subsistit in Ecclesia catholica".

Mit herzlichen Grüßen verabschiede ich mich nach diesem langen Brief und verbleibe mit besten Grüßen. 
